\title{
A Research on Acoustic Emission Signal Source Localization Algorithm
}

\author{
Wenyao Zhu ${ }^{1}$, Jun Zhou ${ }^{2}$, Zemao Zhao ${ }^{1}$ and Yufeng $\mathrm{Wu}^{1}$ \\ ${ }^{1}$ Department of Computer Science, Lishui University, Lishui 323000, China \\ ${ }^{2}$ Logistic Engineering University, Chongqing 401331, China \\ hgzhou2008@163.com
}

\begin{abstract}
To determine the location of the acoustic emission source is one of the main purpose of the acoustic emission test. The accuracy of positioning reflects the degree of coincidence between the position of the sound source and the source location of the actual active defects. Time difference location method and zone location method are the main methods of acoustic emission source location. However, the time difference positioning method has high reliability, and therefore, most of the tests and acoustic emission instruments use time difference method to locate the source of acoustic emission signal. Three point time difference position method and the non-iterative multipoint position method are employed to locate experiment to different types of acoustic emission signals collected. Based on the positioning results, three point time difference positioning method and the non-iteration multipoint positioning method can better position acoustic emission source. However, when the sensor receives the small signal time difference, three point time difference positioning method has large error. The non-iteration multipoint positioning method has positioning advantage when more than four sensors can receive an event signal.
\end{abstract}

Keywords: acoustic emission signal, source localization, Three point time difference position, non-iterative multipoint position

\section{Introduction}

Auxiliary decision-making plan maintenance method by acoustic emission signals of detecting tank corrosion has been widely used in tank repairing. It is necessary to adopt the methods for production halts of the oil tank, to pour out the oil for the room, to open tank for cleaning and testing, which will produce a waste of resources to the oil tank in good condition of tank bottom. Before that, Acoustic emission testing evaluation is done to oil tank to find really oil tank needing shutdown and maintenance to avoid unnecessary waste of resources and huge losses caused by leak detection [1]. The biggest advantage of this way on-line acoustic emission detection is to solve the contradiction between production halts caused by opening tank and storage tank benefits. Acoustic emission testing and acoustic emission signal processing are to obtain the acoustic emission source information as much as possible, and then get the related information of the same materials or structural damages. However, the acoustic emission signal waveform is complicated, which is output by acoustic emission sensors, due to a variety of influential factors such as diversity, sudden and uncertainty of acoustic emission source, the propagation path from acoustic emission source to the sensor, the characteristics of the transducer and acoustic emission instrument. Even there is a big difference in real acoustic emission source. Therefore, in the history of acoustic emission testing technology, a variety of possible signal processing methods have been tried.

Received (February 26, 2018), Review Result (May 20, 2018), Accepted (May 25, 2018) 
In the 1980s, British Fawley refinery plant used acoustic emission technology to inspect and research large-scale atmospheric tank on line for the first time. At present, the acoustic emission technology have become mature and widely used in all kinds of pressure vessel, pressure pipes in the petrochemical industry and marine oil platform test and structure integrity evaluation. The acoustic emission test of oil storage and transportation equipment like the atmospheric storage tank bottom, all kinds of valve and pump, still keeps in applied research stage. The biggest advantage of acoustic emission test has rapid speed and low cost. Therefore, turbulent flow noise caused by load changes leakage, the signals produced by deformation in corrosion thinning area or the acoustic emission signal caused by corrosion layer peeling and cracking, are used to determine whether there is leakage at the tank bottom source (or potential leakage source - local corrosion) and its location. Then, overall state of corrosion of tank bottom makes real-time preliminary judgment, based on the local ultrasonic thickness results of tank wall or roof. Finally, a comprehensive safety assessments made to the integrity of the tank. Acoustic emission signals of the detection to determine tank corrosion tank maintenance plan and auxiliary decision-making method has been widely used in tank repair. The test method like the oil tank halt production, pour the oil to give the empty, and open the tank to clean is adopted, which will produce a waste of resources for tank bottom in good condition of oil tank. Before this, the acoustic emission test to the oil tank is necessary to judge whether it needs maintenance or not and to find oil tank which need real shutdown of maintenance, in order to avoid unnecessary waste of resources and huge losses caused by leak detection. The biggest advantage of this way of on-line acoustic emission detection is to solve the contradiction between halt production and storage oil benefits of oil tank.

Experts and scholars from all over the world have done considerable number of studies on the acoustic emission signal detection of oil tank, from the earliest independent threshold collection, modal analysis and parameters analysis [2], neural network [3] to the subsequent wavelet analysis and wavelet packet analysis [4]. For many years, a great deal of progress has been made in all aspects such as the analysis and processing of actual detection of acoustic emission signal, acoustic emission theory research and perfect operational procedures, etc.,. Wavelet analysis and wavelet packet analysis have strong ability of frequency domain decomposition and waveform reconstruction, which is an irreplaceable calculation tool in signal analysis. Based on neural network pattern recognition technology, wavelet and wavelet packet technology applied in defect signal analysis has obtained the good experimental effect [5].

To identify the place of the acoustic emission source is one of the main purpose of the acoustic emission testing, which is an important indicator to evaluate acoustic emission instrument. The accuracy of positioning reflects the conform degree of between detecting position of the sound source and the actual defect source location. How to improve the positioning precision of the sound source, and minimize leakage location and pseudo positioning, is an important problem in the acoustic emission testing and evaluation as well as the performance of the acoustic emission testing instrument. At present, according to the type of acoustic emission signal, people study many positioning methods. The common type emergency signals of the engineering usually use the regional positioning method and the time difference location method. Regional positioning method has no special requirement to the sensor location, but it requires the sound source signal in the detection area to be received by at least one sensor. The position of the sound source is the sensor nearby location first to receive sound source signal. The advantage of this method is the flexible sensor placement, and the wide detection range, but it can detect the location of sound source only shows a certain area, which is uncertain

Time difference method is a kind of acoustic emission source locating method [6]. Time difference method mainly uses the principle of triangulation. For a determined sound source, the sound source can be located by calculating the distance difference between fixed sound source and three probes. The sound source fixed by this method is a certain point, 
with high reliability. Most of the tests and acoustic emission instruments use time difference to locate the sound source.

Liu Fujun et al., in Zhejiang university chemical machinery research institute, analyzed the acoustic signal amplitude distribution, count distribution, energy distribution, the duration distribution and the rise time distribution based on time difference method, through different defect inspection of the simulation tank bottom plate. According to five kinds of acoustic signal parameters, they determined the effectiveness of the sound source signal, and found that the acoustic signal distribution of acoustic emission detection was consistent with the actual short board.

It is hoped that acoustic emission signals can be obtained to reflect tank floor corrosion situation. However, the environmental noise in the actual detection is quite complicated. Mechanical friction, welding residual stress release, leakage, oxide skin peeling, electronic noise, the birds' chirp, the wind sound, the pipeline vibration, the condensed water and many other factors can interfere with the acoustic emission test. How to exclude noise from the received voice signal is a hot and difficult problem in tank bottom acoustic emission research.

Based on the actual field testing, acoustic emission testing directly positioning function is still limited, and positioning effect is not ideal. The acoustic emission test results and open tank test results still exist certain differences even leakage inspection by mistake. The reliability of acoustic emission testing remains to be improved. In addition, the current national standards only stipulate the event counting to show floor corrosion level [7] from the macroscopic and overall evaluation to the tank bottom. Tank bottom acoustic emission testing technology is not mature, which need stimulate an amount of practical experience and the signal data. It is necessary to further study the corresponding relationship between signal characteristics and tank bottom defect state, and the corresponding detecting operating standards also need to further improve [8].

\section{Acoustic Emission Signal Analog Acquisition}

\subsection{Condensation Acoustic Emission Signal Analog Acquisition}

The acoustic emission scene testing is disturbed by many factors, and experience has shown the most interference from the roof. Vertical storage tank has a certain space between the oil level and the roof. The space is full of oil volatilization and gas as well as the air of the tank size continuously breathing into inside. The oil gas and air cool, form droplets and fall back into the oil level in the roof surface. Droplets noises are transmitted through the oil product directly to the sensor, causing interference to the acoustic emission detection signal. Floating roof tank roof corrosion signal can be transmitted through oil product and reach the sensor, which cause the interference to the acoustic emission signal [9]. Interference signals are mainly condensate water signal. Therefore, the condensation water acoustic emission signal become the typical signal to influence acoustic emission signal of the storage tank. The condensing water test is used to simulate condensing water signal as disturbed signal. The dripping speed is about $1 \mathrm{hit} / \mathrm{s}$; sampling frame length is 8192 , the sampling frequency is $2 \mathrm{MHz}$ Condensation acoustic emission signal simulation system is made of the tank simulation bottom, the sound source excitation device and signal acquisition device, as shown in Figure 1. Condensation acoustic emission signal is shown in Figure 2. 


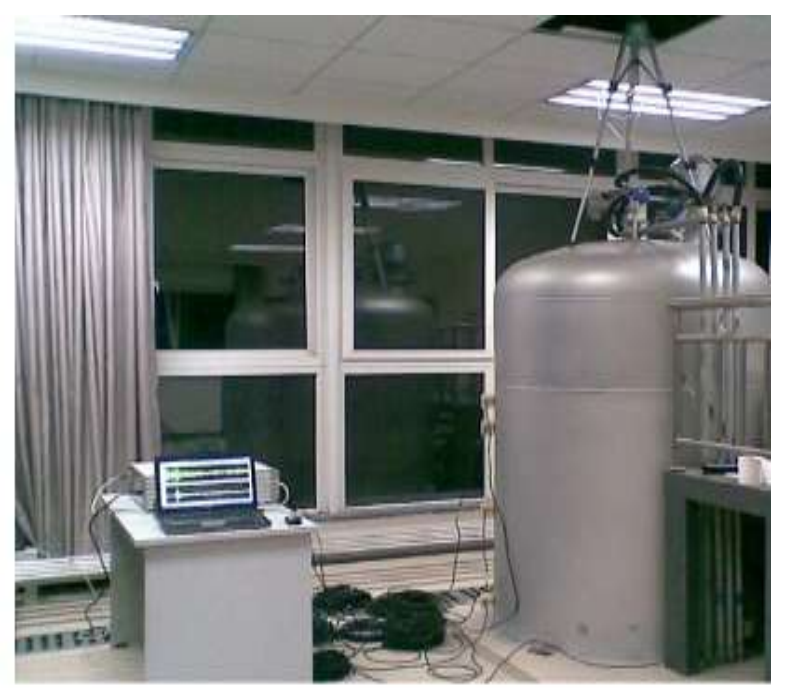

Figure 1. Simulate Collection for Condensation AE Signal

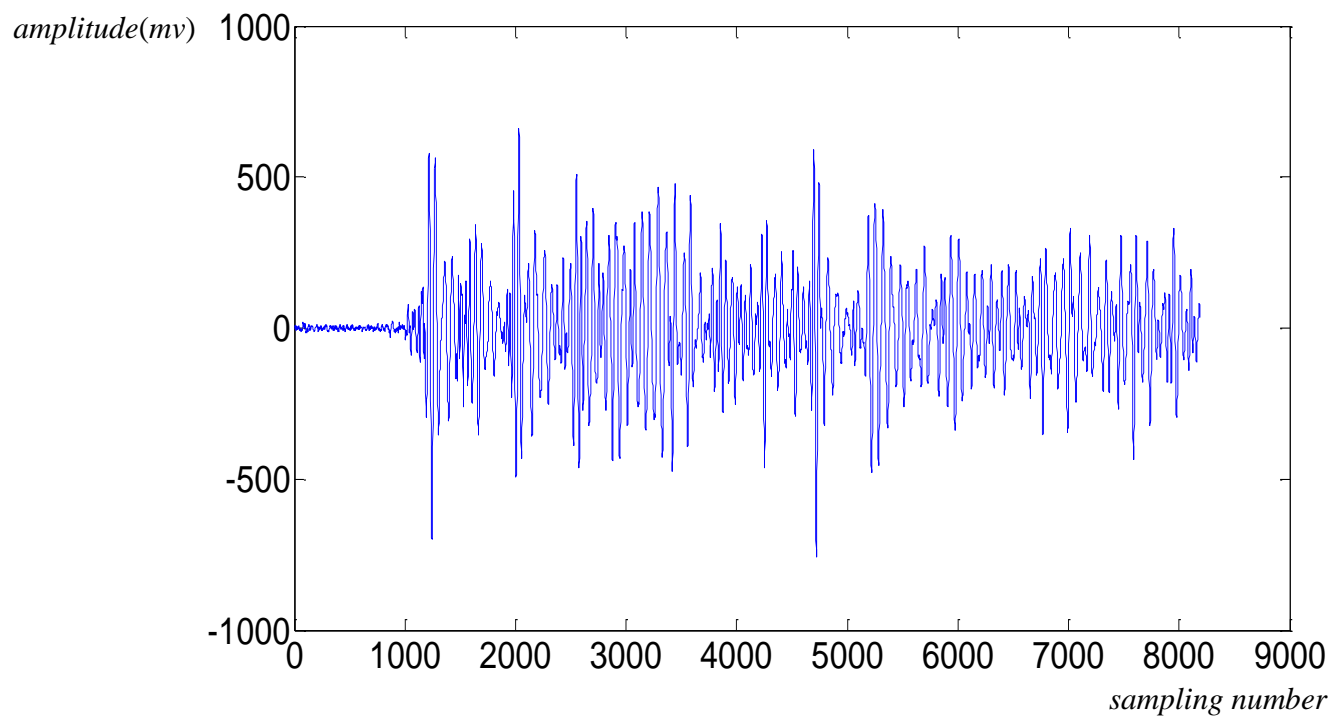

Figure 2. Condensation AE signal

\subsection{Corrosion Acoustic Emission Signal Analog Acquisition}

According to the corrosion mechanism, corrosion can be divided into two categories, including chemical corrosion and electrochemical corrosion. Chemical corrosion refers to a kind of corrosion caused by direct chemical effect of the metal and medium (such as dry oxygen in the air, no water sulfur in the petroleum products, etc.,) such as the corrosion of iron at high temperature, oil storage container by corrosion of oil products with sulfur. Chemical etching is subject to the basic rule of multiphase reaction pure chemical kinetics. There are no electricity generated during corrosion. Electrochemical corrosion refers to a kind of the corrosion phenomena caused by the potential difference of the metal under the condition of the presence of electrolytes (water) or contact with other substances, such as storage tank rusting in the humid air, the corrosion of engine exhaust system due to moisture condensation [10]. Electrochemical corrosion obeys the electrochemical kinetics, and when the corrosion happens, the current is often produced.

The tank bottom surface of storage tanks is generally embalmed, and coated with anticorrosive coating. The coating is damaged over time, so that the surface contacts with 
the water of the tank bottom and bottom impurities. The tank bottom water becomes the electrolyte, and most of the surface corrosion belongs to electrochemical corrosion. The floor surface directly contacts with the tank base, and the corrosion of undersurface is complex, so that electrochemical corrosion, soil corrosion are likely to happen. But the vast majority of floor surface corrosion belongs to electrochemical corrosion. Therefore, the electrolyte corrosion of tank bottom simulation method is used to simulate the tank floor corrosion, and collected acoustic signals mainly characterized as the tank floor corrosion.

Test instrument: acoustic emission detector, detector, common cable. Simulated tank: inner diameter is $600 \mathrm{~mm}$; corrosive liquid is $5 \%$ salt water and liquid level height is 510 $\mathrm{mm}$; liquid mass is $144 \mathrm{~kg}$ with $\mathrm{NaCl} 7.2 \mathrm{~kg}$.

A piece of board of simulation tank bottom is cut off from real tank bottom without the weld. Simulation tank structure is shown in Figure 3. Corrosion acoustic emission signal is shown in Figure 4.

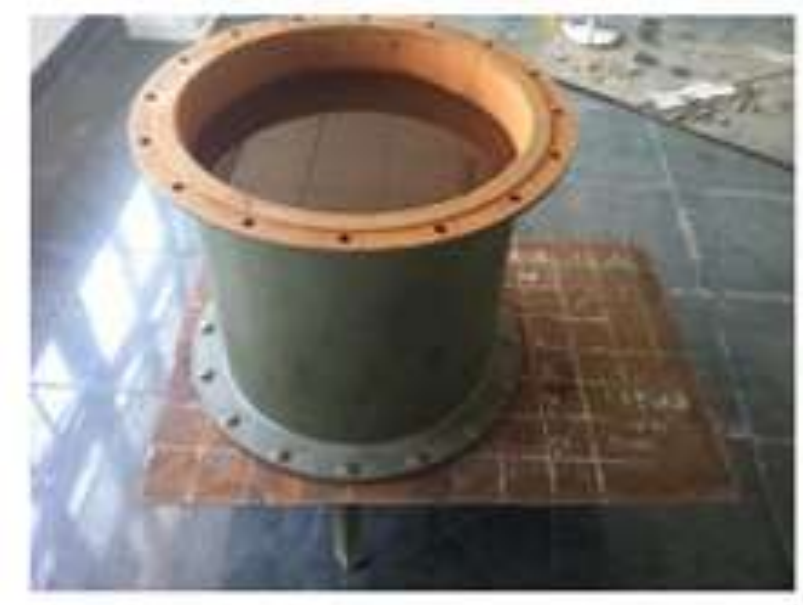

Figure 3. Simulation of Brine Rust Equipment

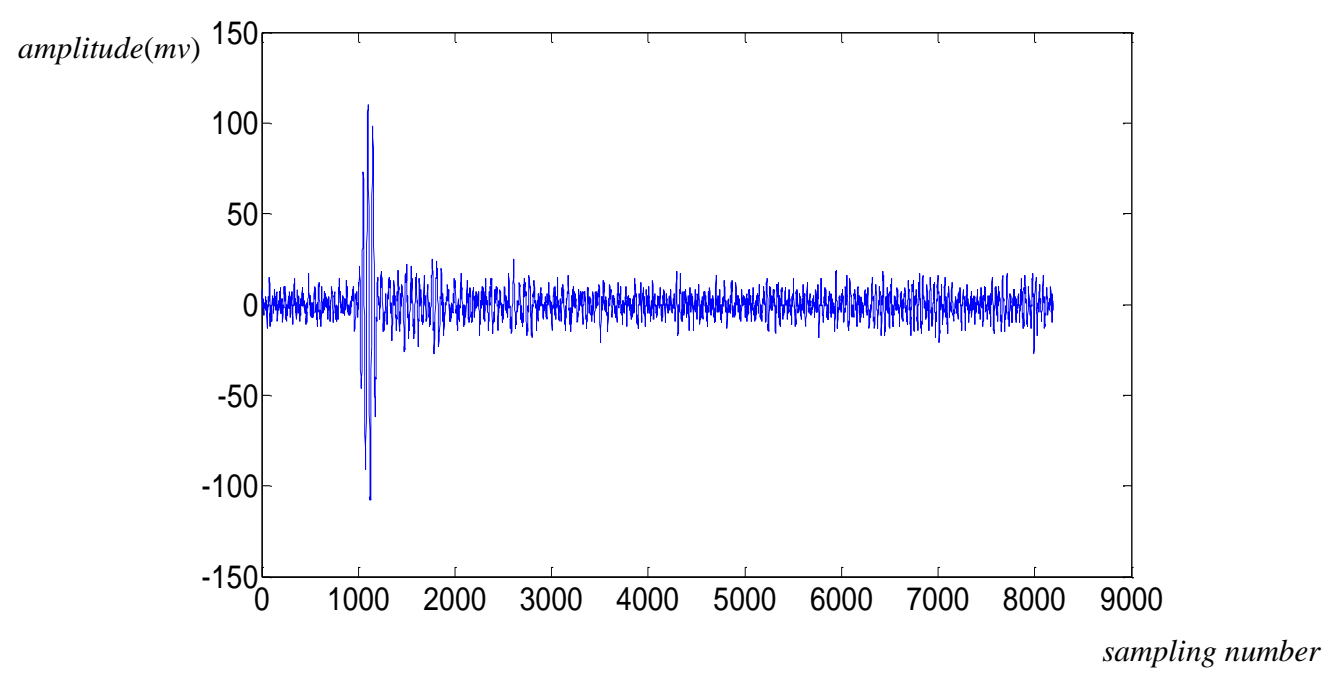

Figure 4. Corrosion AE signal

\subsection{Crack Acoustic Emission Signal Analog Acquisition}

The Nielsen - Hsu broken lead method [11] is used to simulate sheet metal crack acoustic emission signal. The broken lead is often used to calibrate the probe position and measure material attenuation characteristics of the acoustic emission. The specific operation of Nielsen - Hsu broken lead method is shown as follows: HB type automatic lead corewith a 
diameter of $0.5 \mathrm{~mm}$ is used to adjust the length of $2.5 \mathrm{~mm}$ lead center. Along the metal board face, 30 degrees slope breaks the lead. Crack acoustic emission signal is shown in Figure 5.

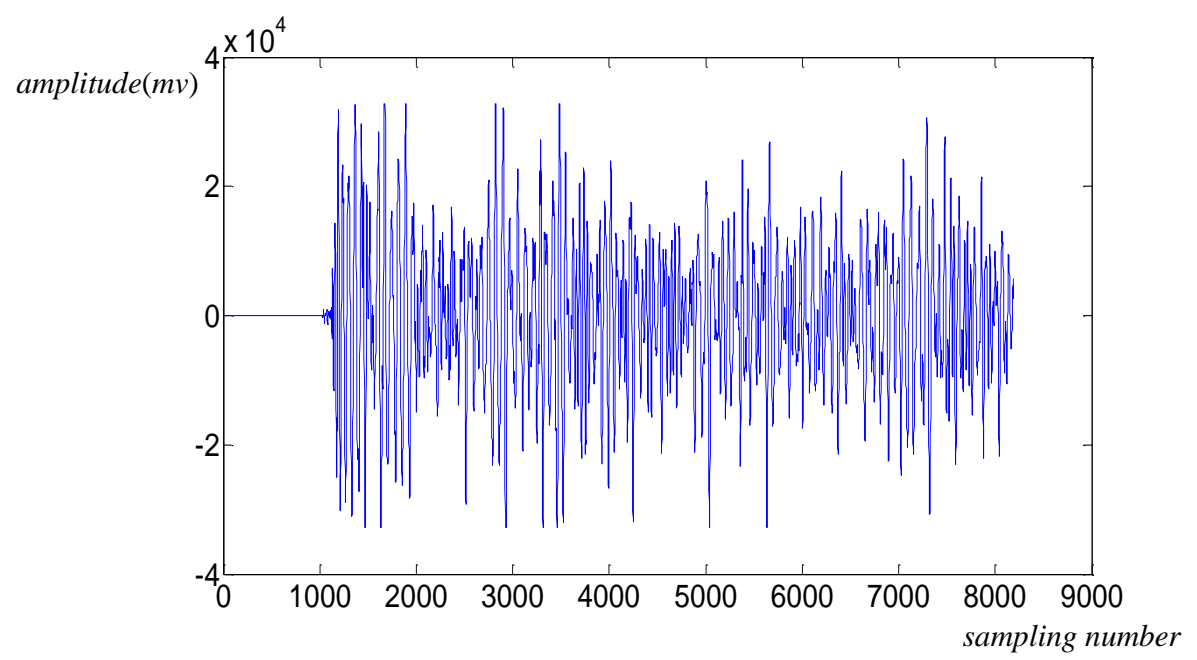

Figure 5. Crack AE Signal

\section{Acoustic Emission Signal Source Localization Principle}

Tank acoustic emission location principle [12]: the defect signals of tank bottom in a very small time difference (the sound source and the probe distance difference and stress wave velocity) are received in three or more probes. According to the time difference of the sensor to receive and relative position sensor, the signal source is positioned as shown in Figure 6:

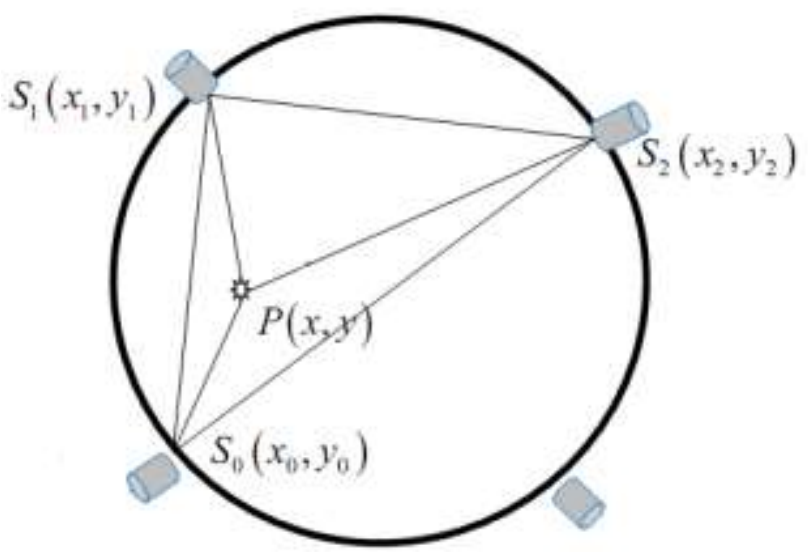

Figure 6. Acoustic Emission Orientation Principle

The planar triangulation algorithm is used as shown in Figure 3.9: any three sensors form a triangle, respectively $S_{0}(0,0)$ (set as coordinate system origin), $S_{2}\left(x_{2}, y_{2}\right)$ and $S_{1}\left(x_{1}, y_{1}\right)$ to determine the position sensor as known, and $P(x, y)$ for the unknown source is solved [13].

$r_{0}, r_{1}, r_{2}$ is set as the distance of the signal source $P$ to $S_{0}, S_{1}, S_{2}$ three sensors.three sensors measuring signal time is respectively $t_{0}, t_{1}, t_{2}$. sound velocity $v$ is set up as knows, $\delta_{1}=r_{1}$ $r_{0}=\left(t_{1}-t_{0}\right) v ; \delta_{2}=r_{2}-r_{0}=\left(t_{2}-t_{0}\right) v ;$ system of equations are shown:

$$
\begin{gathered}
x^{2}+y^{2}=r^{2} \\
\left(x-x_{1}\right)^{2}+\left(y-y_{1}\right)^{2}=\left(r+\delta_{1}\right)^{2}
\end{gathered}
$$




$$
\left(x-x_{2}\right)^{2}+\left(y-y_{2}\right)^{2}=\left(r+\delta_{2}\right)^{2}
$$

Formula (1) is put respectively formula(2), formula (3), $S_{0}(0,0)$ is set as the origin of the polar coordinates, and under the polar coordinate $x=r \cos \theta, y=r \sin \theta$ is solved:

$$
\begin{aligned}
& r\left(x_{1} \cos \theta+y_{1} \sin \theta+\delta_{1}\right)=x_{1}^{2}+y_{1}^{2}-\delta_{1}^{2} \\
& r\left(x_{2} \cos \theta+y_{2} \sin \theta+\delta_{2}\right)=x_{2}^{2}+y_{2}^{2}-\delta_{2}^{2}
\end{aligned}
$$

Set

$$
\begin{aligned}
& A_{1}=x_{1}^{2}+y_{1}^{2}-\delta_{1}^{2} \\
& A_{2}=x_{2}^{2}+y_{2}^{2}-\delta_{2}^{2}
\end{aligned}
$$

Get

$$
r=\frac{A_{1}}{2\left(x_{1} \cos \theta+y_{1} \sin \theta+\delta_{1}\right)}=\frac{A_{2}}{2\left(x_{2} \cos \theta+y_{2} \sin \theta+\delta_{2}\right)}
$$

Set

$$
B=\sqrt{\left(A_{1} x_{2}-A_{2} x_{1}\right)^{2}+\left(A_{1} y_{2}-A_{2} y_{1}\right)^{2}}
$$

To further solve and get:

$$
\begin{gathered}
\cos (\theta-\phi)=k \\
k=\frac{A_{2} \delta_{1}-A_{1} \delta_{2}}{B} \\
\tan \phi=\frac{A_{1} y_{2}-A_{2} y_{1}}{A_{1} x_{2}-A_{2} x_{1}}
\end{gathered}
$$

$\tan \emptyset$ can be measured by the sensor location coordinates and the acoustic emission signal arrival time difference and propagation velocity is obtained, so the $\varnothing$ in $[-\pi, \pi]$, range can be determined. Assume that $B$ is positive value, $\theta$ in $[-\pi, \pi]$ has two solutions. For efficient solution, it must be ensured that the $r$ values have been positive, and sound source location can be determined at this time. Localization algorithm of time difference is a more mature algorithm. Some scholars put forward a new algorithm based on it. This research can use a variety of algorithms to modify signal positioning, and to improve the reliability of the results.

The iterative calculation speed and the acoustic emission positioning algorithm of the target position [14].

The description of sound source location is like the above, all sensor coordinate $S_{0}(0,0)$ is treated as the origin; the $i-1$ sensor $S_{i}\left(r_{i}, \theta_{i}\right)$; the time difference between signals reaching the $i-1$ sensor and reaching the origin sensor is $\underline{t}$, to solve velocity $v, r$, and $\theta$.

(1) Based on TOBIAS algorithm, the composition of equations is shown as follows

$$
2 R v t_{i}+v^{2} t_{i}^{2}+2 R r_{i} \cos q_{i} \cos q+2 R r_{i} \sin q_{i} \sin q=r_{i}^{2}, i=1,2, \cdots, m-1
$$

(2) Transposition changes into matrix form: 


$$
\left(\begin{array}{ccc}
t_{1} & r_{1} \cos q_{1} & r_{1} \sin q_{1} \\
t_{2} & r_{2} \cos q_{2} & r_{2} \sin q_{2} \\
\ldots & \ldots & \ldots \\
t_{m-1} & r_{m-1} \cos q_{m-1} & r_{m-1} \cos q_{m-1}
\end{array}\right)\left(\begin{array}{l}
2 R v \\
2 R \cos q \\
2 R \sin q
\end{array}\right)=\left(\begin{array}{l}
r_{1}^{2} \\
r_{2}^{2} \\
\ldots \\
r_{m-1}^{2}
\end{array}\right)-\left(\begin{array}{l}
t_{1}^{2} \\
t_{2}^{2} \\
\ldots \\
t_{m-1}^{2}
\end{array}\right) v^{2}
$$

Set

$$
A=\left(\begin{array}{ccc}
t_{1} & r_{1} \cos q_{1} & r_{1} \sin q_{1} \\
t_{2} & r_{2} \cos q_{2} & r_{2} \sin q_{2} \\
\ldots & \ldots & \ldots \\
t_{m-1} & r_{m-1} \cos q_{m-1} & r_{m-1} \cos q_{m-1}
\end{array}\right)
$$

Joint orientation matrix.

(3) When $m=4$, solutions of linear equations

$$
\left(\begin{array}{l}
2 R v \\
2 R \cos q \\
2 R \sin q
\end{array}\right)=A^{-1}\left(\begin{array}{l}
r_{1}^{2} \\
r_{2}^{2} \\
\cdots \\
r_{m-1}^{2}
\end{array}\right)-A^{-1}\left(\begin{array}{l}
t_{1}^{2} \\
t_{2}^{2} \\
\cdots \\
t_{m-1}^{2}
\end{array}\right) v^{2}=\left(\begin{array}{l}
a_{1} \\
a_{2} \\
a_{3}
\end{array}\right)-\left(\begin{array}{l}
b_{1} \\
b_{2} \\
b_{3}
\end{array}\right) v^{2}
$$

When $m>4$, The least-square solutions of linear equations is shown:

$$
\left(\begin{array}{l}
2 R v \\
2 R \cos q \\
2 R \sin q
\end{array}\right)=B\left(\begin{array}{l}
r_{1}^{2} \\
r_{2}^{2} \\
\cdots \\
r_{m-1}^{2}
\end{array}\right)-B\left(\begin{array}{l}
t_{1}^{2} \\
t_{2}^{2} \\
\cdots \\
t_{m-1}^{2}
\end{array}\right) v^{2}=\left(\begin{array}{l}
a_{1} \\
a_{2} \\
a_{3}
\end{array}\right)-\left(\begin{array}{l}
b_{1} \\
b_{2} \\
b_{3}
\end{array}\right) v^{2}
$$

Where $B=\left(A^{\mathrm{T}} A\right)^{-1} A^{\mathrm{T}}, A^{\mathrm{T}}$ is $A$ transposed matrix

(4) According to $R v, R \cos q, R \sin q$ and $v^{2}$ the natural constraints between the four variables:

$$
(R v)^{2}=\left((R \cos q)^{2}+(R \sin q)^{2}\right) v^{2}
$$

Put into $R v, R \cos q, R \sin q$, and change standard one unknown variable three times equation:

$$
a x^{3}+b x^{3}+c x+d=0
$$


Where

$$
\left(\begin{array}{l}
a \\
b \\
c \\
d
\end{array}\right)=\left(\begin{array}{c}
b_{2}^{2}+b_{3}^{2} \\
-\left(2 a_{2} b_{2}+2 a_{3} b_{3}+b_{1}^{2}\right) \\
a_{2}^{2}+a_{3}^{2}+2 a_{1} b_{1} \\
-a_{1}^{2}
\end{array}\right)
$$

The solution formula of one unknown variable three times equation can obtain $x=v^{2}$ three solutions. Put into the formula (16) and (17) to compute $r$ and $\theta$. Algorithm can obtain three solutions. According to the physical concept, it can only keep real physical significance and $v>0, \mathrm{r} \geq 0$ real solution.

\section{The Results and Analysis of the Experiment}

Test instrument: Vallen AMSY - 5 acoustic emission detector, Vallen probes, ordinary cable, and test plate is $1500 \times 1200 \times 10 \mathrm{~mm}$ composite defects plate. The probe layout is shown in the figure below, including solid dots for the hole. According to acoustic emission standards, the system performance validation uses $3 \sim 5 \mathrm{~mm}$ cartridge broken lead induced stress wave, and broken pencil lead analog sound source is shown in Figure 7.

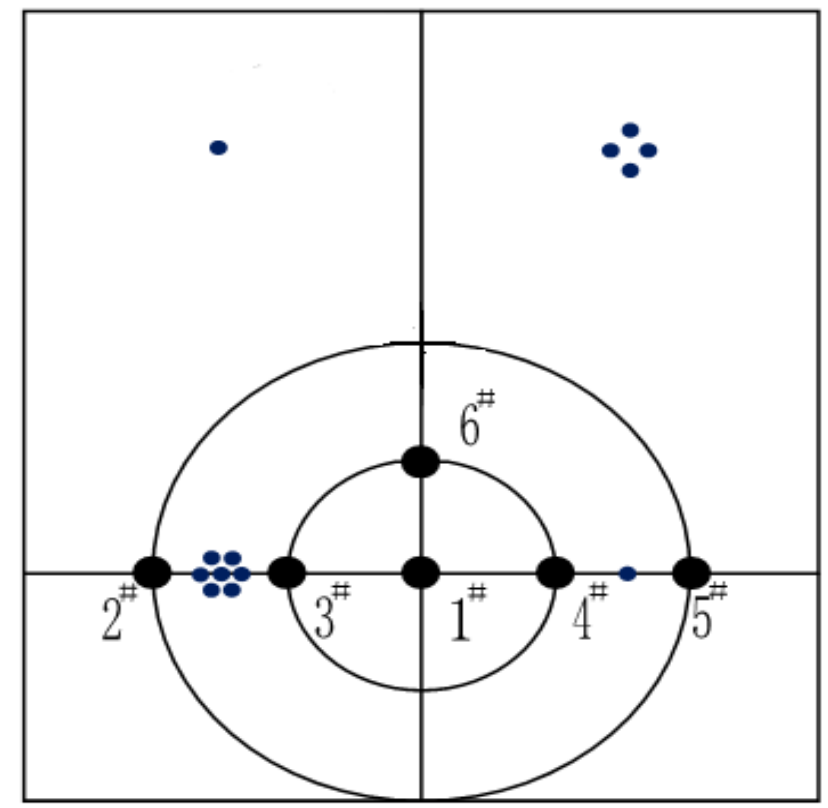

Figure 7. Analogy Experiment of Breaking Lead

Experiment is based on the order of $1 \#$ and $6 \#$ for breaking lead in turn. To the condensed water acoustic emission signals collected and the tank bottom corrosion with acoustic emission signal, three point position and the iterative multipoint positioning algorithm calculate the position as shown in Figure 8. 


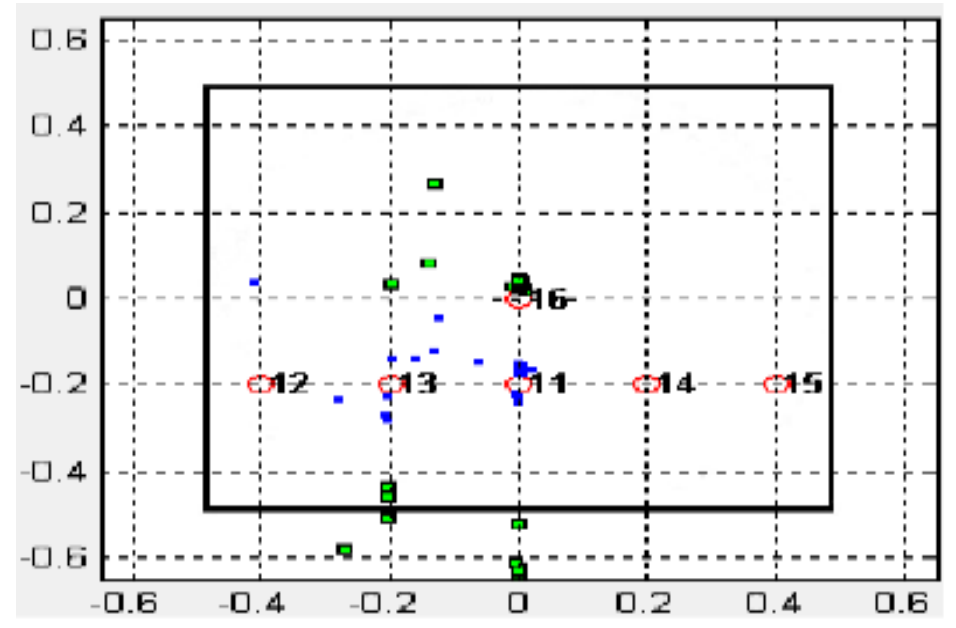

(a)1 \# Positioning Result

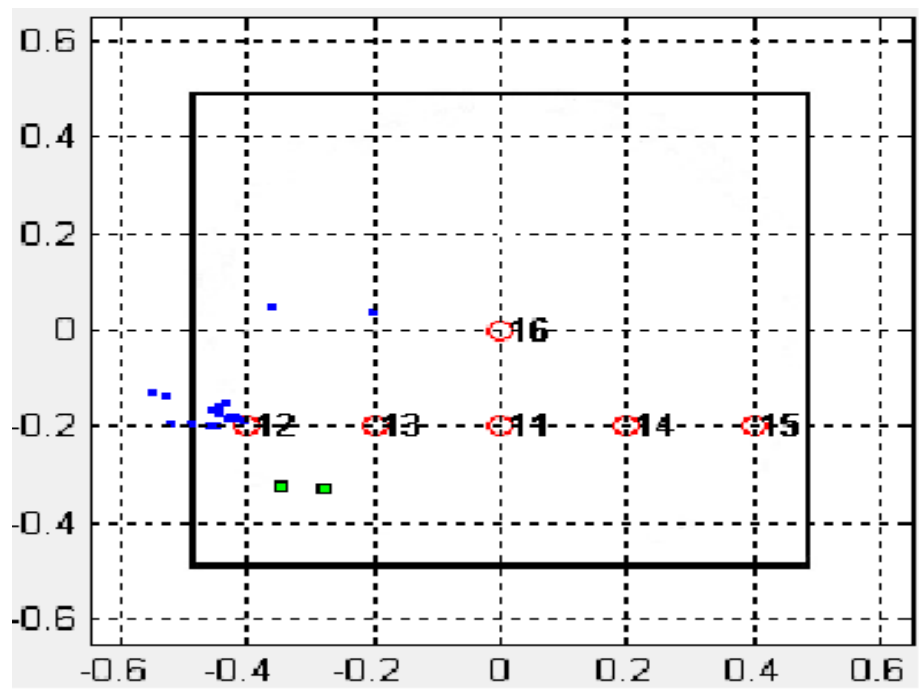

(b)2 \# Positioning Result

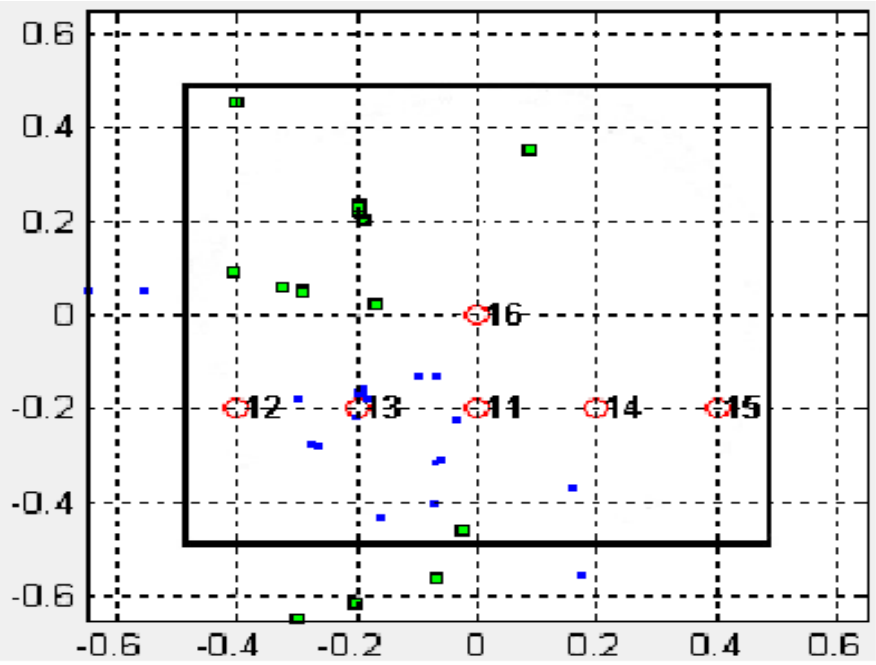

(c)3 \# Positioning Result 


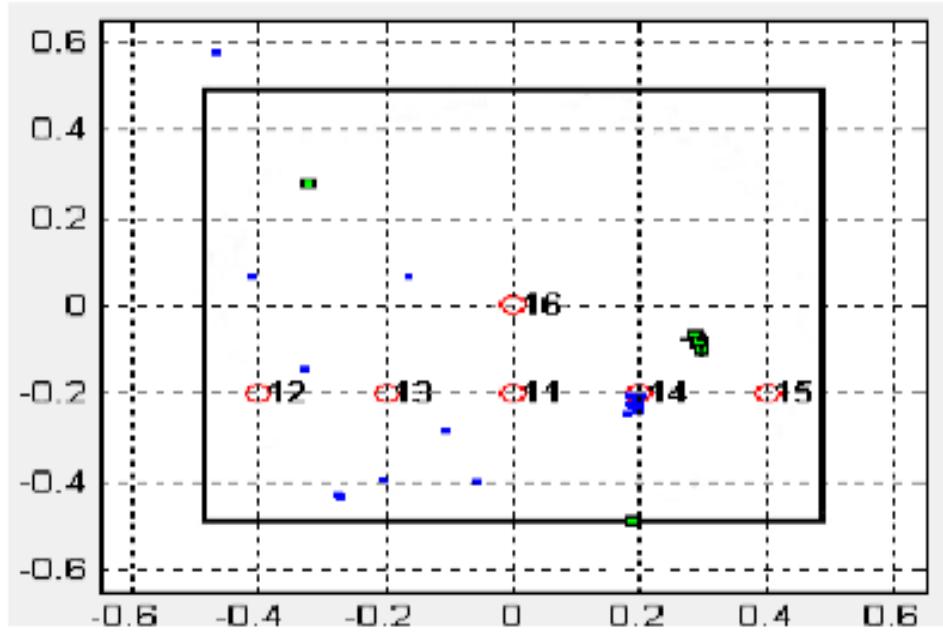

(d) 4\# Positioning Result

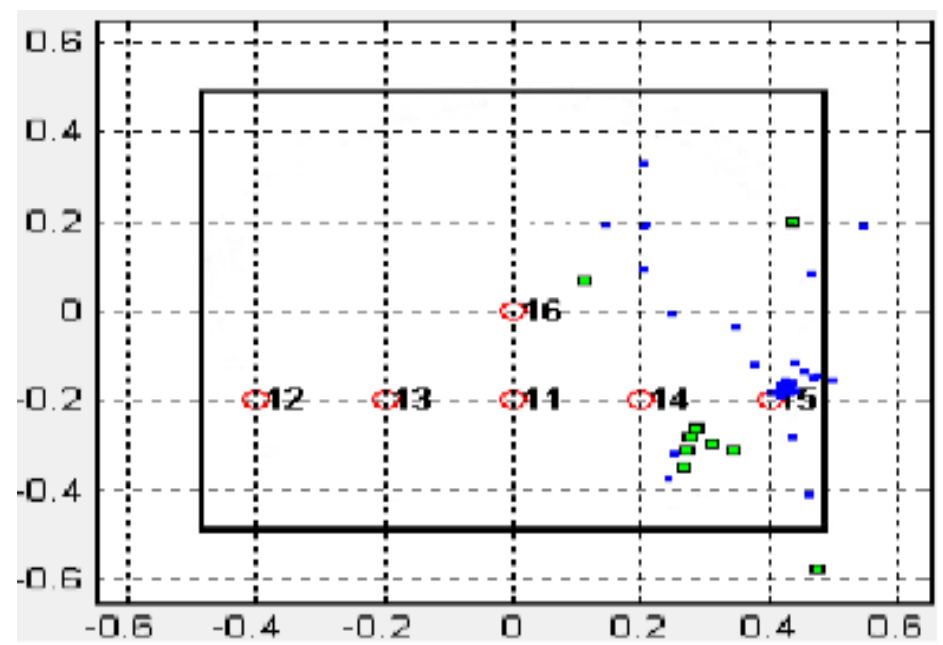

(e) 5\# Positioning Result

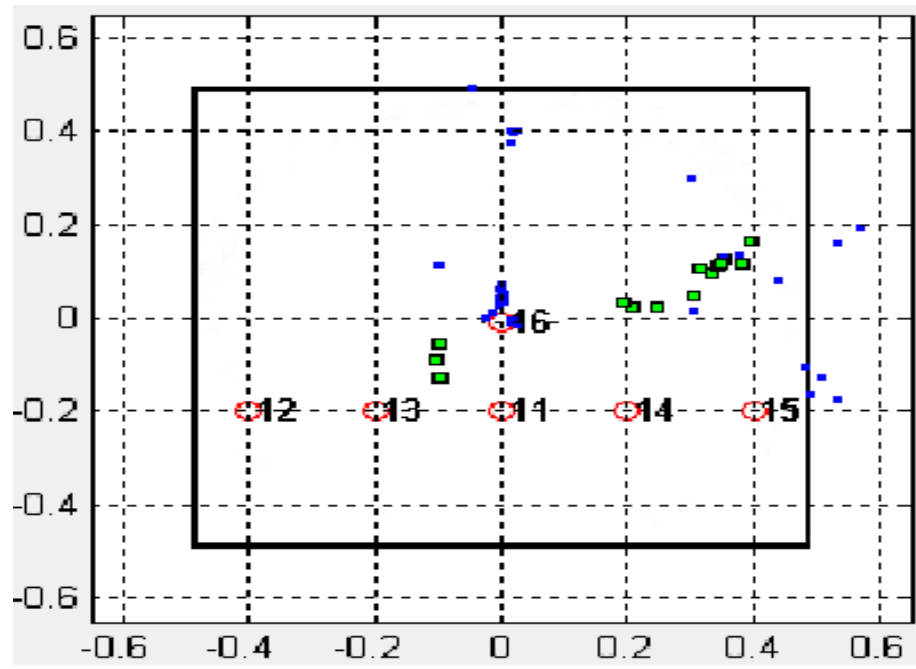

(f) 6\# Positioning Result

Figure 8. Signal Orientation of Test 
In Figure 8, (a) to (f) figures refers to $1 \#$ to 6 \# respectively probe near broken lead acoustic emission location renderings. Solid dot is multipoint positioning legal sites for the iteration, while hollow dot is localization point of time difference of three points. The figure shows that when the probe receives very small time difference, the error of three point time difference positioning method is large. Both methods can identify the source signal. But no iterative multipoint positioning method must be greater than all four sensors to receive an event signal to locate.

\section{Conclusion}

Time difference method is a kind of acoustic emission source locating method. Time difference method mainly uses the principle of triangulation. For a determined sound source, the sound source can be located by calculating the distance difference between fixed sound source and three probes. The sound source fixed by this method is a certain point, with high reliability. Most of the tests and acoustic emission instruments use time difference to locate the sound source. Considering the possibility of actual situation events cannot be received by greater than three sensors at the same time, based on two kinds of typical signal positioning methods to identify the typical signal.

\section{Acknowledgments}

Research supported by Zhejiang Provincial Natural Science Foundation of China under Grand No. Y15F02005.

\section{References}

[1] J. W. Phil Cole, "Acoustic Emission for Corrosion Detection", Middle East Nondestructive Testing Conference \& Exhibition, vol. 3, (2005), pp. 27-30.

[2] L. Songping and C. Jimao, "Modal acoustic emission testing technology", Journal of nondestructive testing, vol. 22, no. 1, (2000), pp. 38-41.

[3] X. Daxue, "The application of Matlab wavelet analysis in signal processing", Science and technology square, vol. 1, (2011), pp. 60-64.

[4] S. Murakami, K. Homma, T. Koike, M. Yamada and S. Yuyama, "AE Source Location Using Neural Network on AE Evaluation of Floor Conditions in Above-Ground Tank", Journal of Solid Mechanics and Materials Engineering, vol. 1, no. 7, (2007), pp. 919-930.

[5] D. Guang, Y. Yongzeng, Z. Ying and Y. Jianglin, "Based on wavelet packet analysis and BP neural network the non-contact acoustic emission of the rolling bearing diagnosis method", Chemical machinery, vol. 36, no. 5, (2008), 274-277294.

[6] L. Wei, D. Guang, L. Feifei and L. Wei, "The development of the atmospheric vertical storage tanks acoustic detector", Journal of daqing petroleum institute, vol. 27, no. 1, (2003), pp. 95-98.

[7] The People's Republic of China, The metal pressure vessel testing and result evaluation method "(JB/T10764-2007). Beijing: The national development and reform commission of the People's Republic of China, (2007).

[8] D. Guang, "Acoustic emission testing technology in China celebrating 30 anniversary of the founding of the Chinese mechanical engineering society NDT branch", Nondestructive testing, vol. 7, (2008), 2008396.

[9] K. Zhongwei, L. Feilu and C. Dixiang, "Based on wavelet transform is the defect database of network storage technology research", Journal of nondestructive testing, vol. 27, no. 10, (2004), pp. 509-513.

[10] L. Li and Z. Deyou, "Jacket offshore platform acoustic emission signal recognition system", Journal of nondestructive testing, vol. 31, no. 1, (2009), pp. 42-45,47.

[11] M. Ji, S. Zhenwei, Y. Guofu and G. Baoqing, "Large rotary ultrasonic on-line detection system", Journa211 of nondestructive testing, vol. 32, no. 3, (2010), pp. 205-205.

[12] N. O. V, B. G. A. and S. A. V., "Calculation of acoustic channel at acoustic-emission testing of steel vertical vessels bottoms", Defektoskopiya, vol. 10, (2003), pp. 55-56.

[13] S. Gongtian, G. Rong-sheng and L. Shifeng, "The parameters of acoustic emission signal analysis method", Journal of nondestructive testing, vol. 24, no. 2, (2002), pp. 72-77.

[14] M. Perrin, L. Gaillet, C. Tessier and H. Idrissi, “Assessment of Stress Corrosion Cracking in Perstressing Srands using AE technique”, Journal of Acoustic Emission, vol. 26, (2008), pp. 32-39. 


\section{Authors}

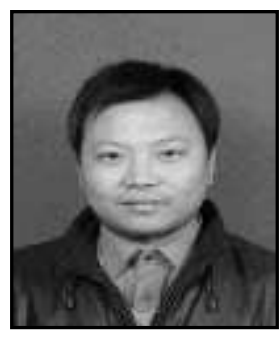

Wenyao Zhu, received his Master Degree from East China Normal University in 2009. He is now a lecturer of Lishui University in China. his research interests include software engineering, pattern recognition, data mining. He has published more than 10 papers on in journals and conferences.

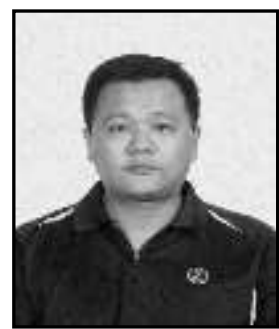

Jun Zhou, received his Doctor Degree from Logistic Engineering University in 2011. He is now a postdoctor of Logistic Engineering University in China. his research interests include image process, pattern recognition. He has published more than 30 papers on in journals and conferences.

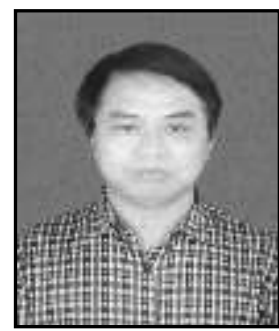

Zemao Zhao, received his $\mathrm{PhD}$ in Computer Science at Nanjing University of Science and Technology in China. He is a Professor of School of Engineering and Design, Lishui University, His research interests include Cryptography and Information Security.

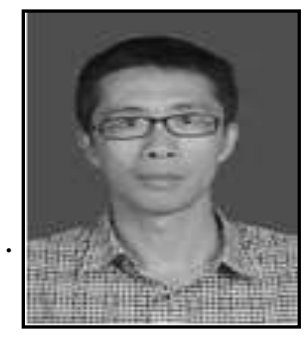

Yufeng Wu, received his Master Degree from Zhejiang University in 2005. He is now an associate professor of Lishui University in China. his research interests include software engineering, pattern recognition, data mining. He has published more than 10 papers in journals and conferences. 
International Journal of Signal Processing, Image Processing and Pattern Recognition Vol. 11, No. 3 (2018) 